\title{
Contents, Vol. 90, 1936
}

\section{Inhaltsverzeichnis.}

Eigenarbeiten.

Seite

Bergler, Karl, Über Kurzwellenbehandlung bei Augen-

erkrankungen 6

Bistis, /., Über einige Fälle von Chininamblyopie ... 13

Fischer, Franz, Beitrag zur Frage der hinteren Ziliar-

nervenschleifen der Sklera 248

Haldimann, Carl, Über Keratitis parenchymatosa bei Lues

congenita der zweiten Generation 146

Heinsius, Ernst, Über schwere Hornhautschädigung durch

konzentriertes Tränengas (Bromazeton) 266

Johansson, Ernst, Impletol in der Ophthalmologie . . . 301

Karsch, Johannes, Kritische Übersicht über die Versuchetherapeutischer Beeinflussung der

Retinitis pigmen-tosa (1877-1935) 157

Kreibig, Wilhelm, Über die amyloide Degeneration der

Bindehaut 129

Kukán, Franz, Ergebnisse der Blutdruckmessungen mit

einem neuen Ophthalmodynamometer 166

Merkulow, 1'., und 5. Minkin, Zur Frage über die Wechsel-beziehung des intrakraniellen und intraokularenDruckes 18

Minkin, S., siehe Merkulow, I.

Niessl v. Mayendor $\uparrow$, E., Über die Existenz angeblicher Er-weichungsherde im Sehnerv, Chiasma und Tractusopticus 241

Nizetic, Z., Fixation des Transplantates bei keratoplasti-schen Eingriffen (nebst Bemerkungen zur Arbeit Friedes „Homo-oder Autokeratoplastik?” Z.f. A. 1935) 305

Römmelt, Joseph, Über das Zusammentreffen einer Conjunctivitis tuberculosa mit dem Auf schießen von Phlyk-tänen 1

Stíel, Andr., Über Probleme der Körnerkrankheit (Trachom) 263

Wünsch-Heitz, Eva, Zur Kenntnis des Einf lusses von druck-

entlastenden Operationen auf das nichtoperierte Auge 259

Verschiedenes.

Dopheide, E., Die Magnetlanze, der Magnetpfriem und die

Magnetpinzette

Johansson, Ernst, Fall von weit vorgeschrittenem Melano-

sarkom 31

Kraupa, E, Albrecht von Graefes Vorlesungen ....

32

-, Megalokornea

192

- IV - 
Seite

Kraupa, E., Staphyloma posticum verum (Graefe) . . . 276

-, Wie die I.Wiener Universitätsaugenklinik in den Besitz des Bildes von J. Barth gelangte und warum Wien zwar die I. Universitätsaugenklinik der Welt, nicht aber den ersten Ordinarius dieses Faches bekam . . 276

-, Eduard von Jägers Ansicht über das Aufstellen von

Theorien zum Selbstzweck 310

- siehe Wokurek, W.

Wokurek, W., und E. Kraupa, Der angeborene Tränennasen-

gangverschluß273

Berichte über die ophthalmologische Literatur.

Linse, Glaskörper, Orbita, Schädel, Nebenhöhlen (Berichtsjahr vom 1. Januar bis 31. Dezember 1935). Von

Dr. Otto ScAmerer-Msixbuxg 34

Glaukom. Von F. Rintelen-Ba, sel 196

Nystagmus (Berichtsjahr 1935). Von Dr. A. Kestenbaum-

Wien 214

Bewegungsapparat des Auges. Von Dr. G. / ancke-Köln 279 Lider, Tränenorgane, Bindehaut

(Berichtsjahr 1935/36). Von

Dr. Peter $\mathrm{S}_{i}$ eg $\phi \mathrm{r}_{i}$-Hamburg

Sehbahn, Auge und Nervenkrankheiten (Berichtsjahr vom1. Januar 1935 bis 31. Dezember

1935). Von WernerKyr ¿eíeis-Ha.mb@ig 350

Gesellschaftsberichte.

51. Zusammenkunft der Deutschen Ophthalmologischen Gesellschaft in Heidelberg am 6., 7.

und 8. Juli in Heidelberg. Referat von

W. Kyrieleis•-Hamburg (Schluß) 58

Verein Rheinisch-Westfälischer Augenärzte. Sitzung vom 24. November 1935 in Köln 117

-. 69. Versammlung. 22. März 1936 in Düsseldorf 227

Augenärztliche Vereinigung des Oberbergamtsbezirks Dortmund.

28. wissenschaftliche Sitzung in der Augenheilanstalt Mülheim-

Ruhr am 15. Februar $1936 \quad 113$

Ophthalmologische Gesellschaft in Wien:

Sitzung vom 16. März 1936 no Sitzung vom 18. Mai 1930223 Sitzung vom 15. Juni 1936290

Diagnose und Therapie 120, 232, 295, 379

Buchbesprechungen 124, 237, 299, 383

In memoriam Dr. Miiller 126

Sundquist $\dagger \quad 240$

S. Sachregister zu Band $90 \quad 384$

Namenregister zu Band $90 \quad 389$ 